Check for updates

Cite this: Soft Matter, 2022, 18,48

Received 15th September 2021, Accepted 24th November 2021

DOI: $10.1039 / \mathrm{d} 1 \mathrm{sm} 01339 \mathrm{~d}$

rsc.li/soft-matter-journal

\section{On the cross-streamline lift of microswimmers in viscoelastic flows $\dagger$}

\author{
Akash Choudhary (D) * and Holger Stark (D) *
}

The current work studies the dynamics of a microswimmer in pressure-driven flow of a weakly viscoelastic fluid. Employing a second-order fluid model, we show that a self-propelling swimmer experiences a viscoelastic swimming lift in addition to the wellknown passive lift that arises from its resistance to shear flow. Using the reciprocal theorem, we evaluate analytical expressions for the swimming lift experienced by neutral and pusher/puller-type swimmers and show that they depend on the hydrodynamic signature associated with the swimming mechanism. We find that, in comparison to passive particles, the focusing of neutral swimmers towards the centerline can be significantly accelerated, while for force-dipole swimmers no net modification in cross-streamline migration occurs.

Biological microswimmers are ubiquitous in polymeric media such as cervical, bronchial, and intestinal mucus films., During the generation of biofilms most bacteria release a mixture of proteins, DNA, and polysaccharides, endowing the fluid with viscoelastic properties, ${ }^{3,4}$ which significantly alter the swimmer's dynamics. ${ }^{5,6}$ Pathogens like ulcer-causing Helicobacter pylori survive in a harsh acidic environment by altering the rheology of the mucus lining in the stomach. ${ }^{7}$ Several theoretical and experimental studies on the dynamics of motile microorganisms in Newtonian flows have revealed their rich dynamics and ability to swim against fluid flows, which aids in seeking nutrients and in reproduction. ${ }^{8-15}$

Although recent works have provided insights into selfpropulsion in non-Newtonian environments, ${ }^{15-32}$ few have studied the impact of fluid rheology on the dynamics of microswimmers in confined flows. ${ }^{33-36}$ Mathijssen et al. ${ }^{33}$ developed a model to predict the dynamical states of microswimmers in non-Newtonian Poiseuille flows. Employing a second-order fluid model, they demonstrated that normal-stress differences

Institute of Theoretical Physics, Technische Universität Berlin, 10623 Berlin, Germany.E-mail: a.choudhary@campus.tu-berlin.de, holger.stark@tu-berlin.de $\dagger$ Electronic supplementary information (ESI) available. See DOI: 10.1039/ d1sm01339d reorient the swimmers to cause centerline upstream migration (rheotaxis). This suggests that non-Newtonian properties can help microorganisms evade the boundary accumulation, prevalent in quiescent Newtonian fluids. ${ }^{37,38}$

The evidence of centerline reorientation of microswimmers can be traced back to pioneering studies on viscoelastic focusing of passive particles in Poiseuille flows. ${ }^{39-41}$ These studies showed that normal stresses exert a lift force that focuses the particles on the centerline. Ho and Leal ${ }^{41}$ used the reciprocal theorem and derived an analytical expression for this lift in weakly elastic Boger fluids. The study suggested that the hydrodynamic disturbances around the particle produce a hoop stress that, in the presence of non-uniform shear rate, generates a cross-streamline lift. Recent progress in electrophoresis ${ }^{42-44}$ has also shed light on the importance of hydrodynamic disturbances in determining these lift forces.

Active microswimmers, as opposed to passive particles, generate additional disturbance flow fields in the fluid due to their self-propulsion. Therefore, the associated lift force or velocity should also have an active component that is characteristic of the self-propulsion mechanism. Since this component is absent in the recently proposed models, ${ }^{33}$ in this communication we derive the 'swimming lift' in a secondorder fluid (SOF), and show how it affects the dynamics of a microswimmer in the Poiseuille flow of a viscoelastic fluid. We choose the SOF model because it provides an asymptotic approximation for a majority of slow and slowly varying viscoelastic flows. $^{45,46}$

Fig. 1 shows a spherical swimmer of radius $a$ at position $r$ that self-propels with velocity $\boldsymbol{v}_{\mathrm{s}}=\boldsymbol{v}_{\mathrm{s}} \boldsymbol{p}$ in a two-dimensional pressure-driven flow $\boldsymbol{v}_{\mathrm{f}}=v_{\mathrm{m}}\left[1-(x / w)^{2}\right] \boldsymbol{e}_{\mathrm{z}}$, where $v_{\mathrm{m}}$ is the maximum flow velocity and $w$ is the half channel width. The flow profile of the second-order fluid is identical to Poiseuille flow but the pressure field varies in the $x$-direction. ${ }^{41}$ In the absence of noise, the swimmer's dynamics is governed by

$$
\dot{\boldsymbol{r}}=\boldsymbol{p}+\overline{\boldsymbol{v}}_{\mathrm{f}}+\mathcal{F}(x, \boldsymbol{p}) \boldsymbol{e}_{x}, \quad \dot{\boldsymbol{p}}=\frac{1}{2}\left(\nabla \times \overline{\boldsymbol{v}}_{\mathrm{f}}\right) \times \boldsymbol{p},
$$




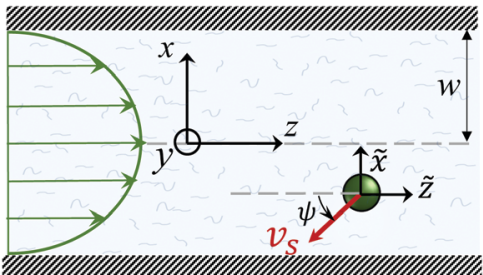

Fig. 1 A spherical microswimmer with velocity $v_{s} \boldsymbol{p}$ moves in a pressuredriven flow of a second-order fluid inside a channel with half width $w$. The coordinate frame $\{\tilde{x}, \tilde{y}, \tilde{z}\}$ co-moves with the swimmer.

where the velocities are non-dimensionalized by swimming speed $\left(\overline{\boldsymbol{v}}_{\mathrm{f}}=\boldsymbol{v}_{\mathrm{f}} / v_{\mathrm{s}}, \bar{v}_{\mathrm{m}}=v_{\mathrm{m}} / v_{\mathrm{s}}\right)$, lengths by $w$, and time by $w / v s . \mathcal{F}$ denotes the total viscoelastic lift velocity, which comprises the passive and swimming lift. Below, we derive the analytical expressions for the lift velocities. We note that normal stresses also modify the particle rotation and the drift velocity along the channel axis. We evaluated these modifications and found that they do not play a significant role in determining the swimmer dynamics.

The inertia-less or creeping flow hydrodynamics is governed by the continuity equations for mass and momentum, which we formulate here in the co-moving swimmer frame $\{\tilde{x}, \tilde{y}, \tilde{z}\}$ as:

$$
\tilde{\nabla} \cdot \boldsymbol{V}=0, \quad \tilde{\nabla} \cdot \mathbf{T}=0
$$

in order to calculate the viscoelastic lift velocity. The length, velocity, and pressure in (2) are non-dimensionalized by $a, \kappa v_{\mathrm{m}}$, and $\mu \kappa v_{\mathrm{m}} / a$, respectively. Here, $\kappa$ is the particle to channel width ratio $(a / 2 w)$ and $\mu$ is the fluid viscosity. In the above equation, $\boldsymbol{V}$ is the total velocity field, $\mathbf{T}$ is the total stress tensor of a second-order fluid and thus has the form: ${ }^{46} \mathbf{T}=-P \mathbf{I}+2 \mathbf{E}+$ WiS. Here, $\mathbf{E}$ denotes the rate of strain tensor, $\mathbf{S}$ the total polymeric stress tensor, and $\mathrm{Wi}=\left(\Psi_{1}+\Psi_{2}\right) G / \mu$ is the shear based Weissenberg number, where $\mu$ is the viscosity, $G=v_{\mathrm{m}} / 2 w$ characterizes the shear rate in the background flow, and $\Psi_{1}, \Psi_{2}$ represents the dimensional steady-shear normal stress coefficients that are measured experimentally. ${ }^{46}$ The polymeric stress tensor $\mathbf{S}=4 \mathbf{E} \cdot \mathbf{E}+2 \delta \stackrel{\Delta}{\mathbf{E}}$ is non-linear in $\mathbf{E}$ and contains the lower-convected time derivative of $\mathbf{E}$ denoted by $\Delta$. The viscometric parameter $\delta=-\Psi_{1} / 2\left(\Psi_{1}+\Psi_{2}\right)$ generally varies from -0.5 to -0.7 for most viscoelastic fluids. ${ }^{47-49}$

We now focus on determining the lift velocity of a microswimmer that disturbs the background flow in two ways. First of all, the microswimmer resists straining by the flow and second, it generates a flow field characteristic of its swimming mechanism, for which we first take a source-dipole swimmer. We split the total velocity field $\left(\boldsymbol{V}=\boldsymbol{v}^{\infty}+\boldsymbol{v}\right)$ into background flow field $\boldsymbol{v}^{\infty}$ (in our case the Poiseuille flow in the co-moving swimmer frame) and disturbance field $\boldsymbol{v}$, and adopt the same nomenclature for the polymeric stress tensor, $\mathbf{S}=\mathbf{s}^{\infty}+\mathbf{s}$, where $\mathbf{s}$ is the polymeric stress tensor associated with the disturbance flow field. We show its full form in the ESI. $\dagger$ Substituting this in the governing eqn (2) yields the equation for the disturbance field $v$ :

$$
\tilde{\nabla} \cdot \boldsymbol{v}=0, \quad-\tilde{\nabla} \boldsymbol{p}+\tilde{\nabla}^{2} \boldsymbol{v}=-\mathrm{Wi}(\tilde{\nabla} \cdot \mathbf{s})
$$

The methodology of evaluating the lift velocity closely follows the recent analysis of Choudhary et al. ${ }^{43}$ Here, we only summarize the basics and elaborate on details in the ESI. $\dagger$ Assuming weak viscoelasticity (Wi « 1), we perform a perturbation expansion in Wi and only take the first two contributions of eqn (3) in this expansion: the Stokes equation for the zeroth order of the disturbance field, $-\tilde{\nabla} p_{0}+\tilde{\nabla}^{2} \boldsymbol{v}_{0}=0$ and $-\tilde{\nabla} p_{1}+$ $\tilde{\nabla}^{2} \boldsymbol{v}_{1}=-\tilde{\nabla} \cdot \mathbf{s}_{0}$ for the first order. Following earlier works on viscoelastic lift, ${ }^{41,43}$ we use the reciprocal theorem to attain the lift velocity from the first-order problem,

$$
\mathcal{F}=-\frac{\mathrm{Wi}}{6 \pi} \int_{V_{\mathrm{f}}} \mathbf{s}_{0}: \tilde{\nabla} \boldsymbol{u}^{t} \mathrm{~d} V
$$

Here, $\boldsymbol{u}^{t}$ is the auxiliary or test velocity field that belongs to a forced particle moving along the $x$-direction with unit velocity in a Newtonian fluid. The polymeric stress tensor $\mathbf{s}_{0}$ is associated with the Stokes solution $\boldsymbol{v}_{0}$ of the microswimmer. The zeroth order of the disturbance flow field consists of (i) a source-dipole field, which we adopt from the squirmer model, ${ }^{50-52} \boldsymbol{v}_{0}^{\text {swim }}=\frac{\tilde{v}_{\mathrm{s}} \boldsymbol{p}}{2 \tilde{r}^{3}} \cdot\left[\frac{3 \tilde{r} \tilde{r}}{\tilde{r}^{2}}-\mathbf{I}\right]$, and (ii) the passive disturbance field $\boldsymbol{v}_{0}^{\text {passive }}$, where $\tilde{v}_{\mathrm{s}}=v_{\mathrm{s}} /\left(v_{\mathrm{m}} \kappa\right)$. For the latter, the leading contribution is the stresslet, while higher-order terms due to the curvature in the Poiseuille flow profile are obtained from Lamb's general solution. ${ }^{53}$ The bounding channel walls modify these bulk flow fields. However, by using the method of reflections for small particles $(\kappa \ll 1)$, one can show that they do not alter the lift velocity in leading order in $\kappa^{43}$ Therefore, it is sufficient to perform the integration in eqn (4) in the infinite domain. Using the $s_{0}$ corresponding to Stokes velocity field in eqn (4), results in the viscoelastic lift velocity given in units of $v_{\mathrm{s}}$ :

$$
\begin{aligned}
\mathcal{F}(x, \psi) & =\mathcal{F}_{\text {passive }}+\mathcal{F}_{\text {swim }} \\
& =\mathrm{Wi}\left[\frac{80}{9} x \bar{v}_{\mathrm{m}} \kappa^{2}(1+3 \delta)-x(1+\delta) \cos \psi\right] .
\end{aligned}
$$

The first component in eqn (5) is the passive lift $\mathcal{F}_{\text {passive }}{ }^{41}$ By fixing $\delta$ to a widely-used value of -0.5 (i.e. $\Psi_{2}=0$ ), we observe that $\mathcal{F}_{\text {passive }}$ focuses the swimmer towards the centerline. The second component is the swimming lift $\mathcal{F}_{\text {swim }}$ that arises due to the source-dipole disturbance created by the neutral swimmer. We note two striking features of $\mathcal{F}_{\text {swim }}$ : the dependence on swimmer orientation through $\cos \psi$ and that its magnitude is larger by a factor $\kappa-{ }^{2}$ compared to the first term. $\$$

Now, we substitute $\mathcal{F}$ into the dynamic eqn (1) and examine the effect of $\mathcal{F}_{\text {swim }}$ on the microswimmer dynamics. We find two fixed points in the $x-\psi$ plane at $x=0$, with the microswimmer oriented upstream $(\psi=0)$ or downstream $(\psi= \pm \pi)$. A linear stability analysis provides the following eigenvalues for these fixed points:

$$
\begin{aligned}
\lambda_{\text {up }} & \approx \frac{\mathrm{Wi}}{18}\left[-9(1+\delta)+80(1+3 \delta) \kappa^{2} \bar{v}_{\mathrm{m}}\right] \pm i \bar{v}_{\mathrm{m}}{ }^{1 / 2}, \\
\lambda_{\text {down }} & \approx \frac{\mathrm{Wi}}{18}\left[9(1+\delta)+80(1+3 \delta) \kappa^{2} \bar{v}_{\mathrm{m}}\right] \pm \bar{v}_{\mathrm{m}}{ }^{1 / 2} .
\end{aligned}
$$


(a) 1.0

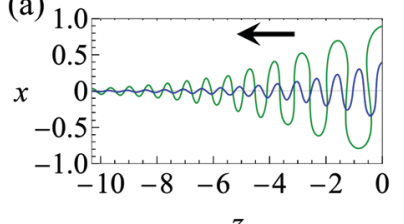

(c) 80

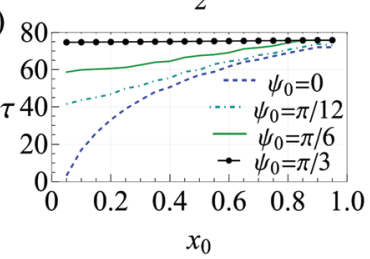

(b)

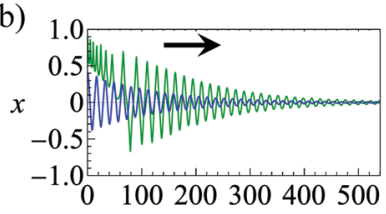

(d)

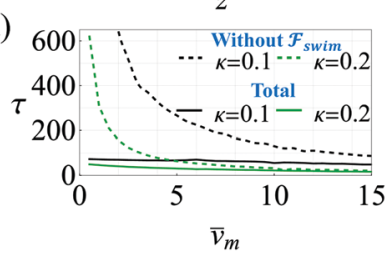

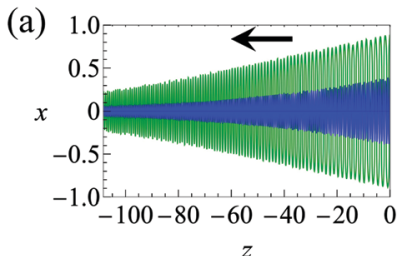

(c)

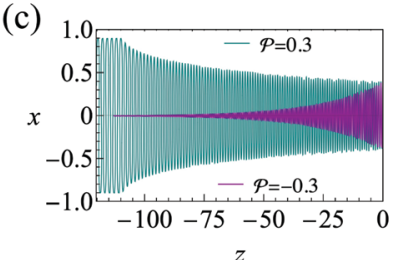

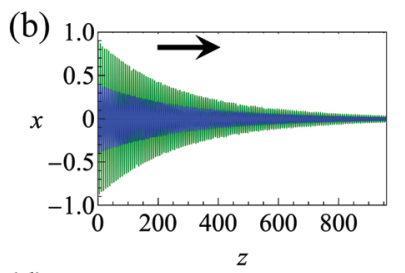

(d)

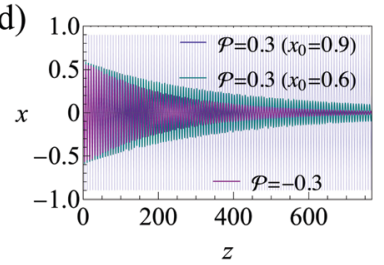

Fig. 2 Trajectories showing the centerline focusing of a neutral (sourcedipole) swimmer oriented upstream while (a) swimming upstream $\left(\bar{V}_{m}=0.9, \leftarrow\right)$ and (b) swimming downstream $\left(\bar{V}_{m}=8, \rightarrow\right)$. The blue and green trajectories only differ in the initial condition $x_{0}$; for both trajectories we choose $\psi_{0}=0$. Other parameters: $\mathrm{Wi}=0.1, \kappa=0.1, \delta=-0.5$. (c) Variation of focusing time $\tau$ for different initial conditions $x_{0}$ and $\psi_{0}$. Parameters: $\bar{v}_{m}=0.9, \mathrm{Wi}=0.1, \kappa=0.1$. Trajectories are considered focused when the amplitude of oscillations reaches $x=0.05$. (d) Focusing time for various flow rates and two different $\mathcal{F}_{\text {swim }}$ swimmer sizes. Dashed lines depict the focusing time in the absence of $\mathcal{F}_{\text {swim }}$. We plot the maximum focusing time that occurs for the initial condition $x_{0}=0.9, \psi_{0}=0$.

For a typical value of $\delta=-0.5$ and weak viscoelasticity limit (Wi $\ll 1$ ), the downstream orientation corresponds to a saddle fixed point (associated with $\lambda_{\text {down }}$ ), while the upstream orientation and swimming along $x=0$ corresponds to a stable fixed point (associated with $\lambda_{\text {up }}$ ). For $\delta=-0.5$, the sign of the real part of $\lambda_{\text {up }}$ shows that both swimming and passive lift components stabilize the upstream orientation. Fig. 2(a) and (b) show the upstream and downstream trajectories of the swimmer. In both cases the swimmer attains the upstream orientation, which is stabilized by both $\mathcal{F}_{\text {passive }}$ and $\mathcal{F}_{\text {swim. }}$. The strong swimming lift helps the neutral microswimmer reach the centerline more rapidly, as suggested by the factor of $\kappa-^{2}$ in eqn (5). To quantify this accelerated focusing, we calculate the focusing time $\tau$ as the time required for the oscillatory amplitudes to decrease to $5 \%$ of the half channel width, i.e., $x=0.05$. For upstream swimming at low flow rate $\left(\bar{v}_{\mathrm{m}}=0.9\right)$, Fig. $2(\mathrm{c})$ shows how the focusing time changes with initial position and orientation. We further evaluate the impact of $\mathcal{F}_{\text {swim }}$ on the focusing time in Fig. 2(d). At low flow rates $\left(\bar{v}_{\mathrm{m}} \sim 1\right)$, the centerline focusing is much faster when $\mathcal{F}_{\text {swim }}$ is taken into account, however, this difference withers as the flow rate increases; this can also be observed from the analytical expression (5). We further analyze the focusing time over a wider range of parameters in the ESI. $\dagger$

Now, we shift our attention from neutral squirmers to flagellated microorganisms, such as E. coli and Chlamydomonas, that generate a force-dipole field at the leading order: ${ }^{37,54}$ $\boldsymbol{v}_{0}=\mathcal{P} \tilde{v}_{\mathrm{s}} \boldsymbol{r}\left[\frac{-1}{r^{3}}+3 \frac{(\boldsymbol{r} \cdot \boldsymbol{p})^{2}}{r^{5}}\right]$. Here $\mathcal{P}$ is the force-dipole strength in units of $8 \pi \mu a^{2} v_{\mathrm{s}}$, which depends on the swimming mechanism. ${ }^{37,55,56}$ Earlier studies on E. coli ${ }^{56,57}$ and Chlamydomonas $^{58}$ suggest that $|\mathcal{P}|$ varies roughly between 0.04 and 0.3 .

Fig. 3 Trajectories showing the centerline focusing of pusher $(\mathcal{P}=0.3)$ in (a) upstream swimming $\left(\bar{v}_{m}=0.9, \leftarrow\right)$ and (b) downstream swimming $\left(\bar{v}_{m}=3, \rightarrow\right)$. The trajectories for a puller are similar. The blue and green trajectories in (a) and (b) only differ in the initial condition $x_{0}$; for both trajectories we choose $\psi_{0}=0$. (c) Upstream $\left(\bar{v}_{m}=0.9\right)$ and (d) downstream trajectories $\left(\bar{v}_{m}=3\right)$ with hydrodynamic wall interactions (8) incorporated. For the initial condition, $x_{0}=0.9$, the blue trajectory in the background of (d) shows downstream swinging across the whole channel cross section. We employ hard-core repulsion at the walls. Other parameters: $\mathrm{Wi}=0.1, \kappa=0.1, \delta=-0.5$.

Following the procedure outlined for a source-dipole swimmer, we obtain the swimming lift velocity of the force-dipole swimmer in the units of $v_{\mathrm{s}}$ as

$$
\mathcal{F}_{\text {swim }}=(8 / 3) \mathrm{Wi} \kappa \mathcal{P}(1+3 \delta) \sin 2 \psi,
$$

and find it to depend on the constant curvature in Poiseuille flow, as detailed in the ESI. $\dagger$ Although this lift is $O\left(\kappa-^{1}\right)$ larger than $\mathcal{F}_{\text {passive }}$, it does not result in net cross-stream migration because it is independent of lateral position. The trajectories in Fig. 3(a) and (b) show the dynamics of a force-dipole swimmer. In the steady state, the swimmer shows a stable upstream orientation and swims along the centerline since a deviation from $\psi=0$ is counteracted by flow vorticity. For $\psi=0, \mathcal{F}_{\text {swim }}$ vanishes and the focusing along the centerline is purely due to $\mathcal{F}_{\text {passive }}$.

So far we have neglected the hydrodynamic interactions of microswimmers with the bounding channel walls. For forcedipole swimmers, the hydrodynamic wall interactions add a modification of order $\kappa^{2}$ and $\kappa^{3}$ to the evolution equations of position and orientation, respectively: ${ }^{11,59}$

$$
\begin{aligned}
& \dot{x}=-\sin \psi+\mathcal{F}-\frac{3 \mathcal{P}\left(3 \sin ^{2} \psi-1\right)}{8} \kappa^{2}\left[\frac{1}{(1-x)^{2}}-\frac{1}{(1+x)^{2}}\right], \\
& \dot{\psi}=x \bar{v}_{\mathrm{m}}-\frac{3 \mathcal{P} \sin 2 \psi}{16} \kappa^{3}\left[\frac{1}{(1-x)^{3}}+\frac{1}{(1+x)^{3}}\right] .
\end{aligned}
$$

Upstream trajectories in Fig. 3(c) closely resemble the behavior reported previously for pure Newtonian fluids. ${ }^{11}$ We observe that the hydrodynamic wall attraction of pushers ${ }^{37}$ overcomes $\mathcal{F}_{\text {passive }}$ and results in swinging across the whole channel cross section, where the strong vorticity near the walls always reorients the swimmer away from it. In contrast, for downstream 

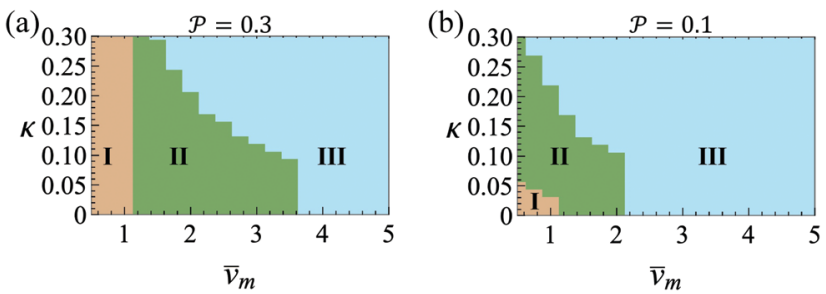

Fig. 4 State diagram for pushers (a) $\mathcal{P}=0.3$ and (b) $\mathcal{P}=0.1$. Region । unstable trajectories that spiral out to result in channel-wide swinging, Region III: stable centerline swimming, Region II: both dynamic states coexist; they depend on the initial condition. Other parameters: $\mathrm{Wi}=0.1$, $\psi_{0}=0$.

swimming at larger flow rates [Fig. 3(d)], $\mathcal{F}_{\text {passive }}$ competes with the hydrodynamic wall interactions, which results in some trajectories focusing at the centerline and some following channel-wide swinging depending on the initial condition. The state diagram in Fig. 4(a) illustrates this behavior of pushers for a wide range of parameters. At low flow rates (region I) hydrodynamic wall interactions dominate and thus channel-wide swinging occurs. As the flow rate increases the system enters region II. Here, the realized trajectories depend on the initial condition as depicted in Fig. 3(d): swimmers starting near the walls follow downstream swinging across the channel cross-section, whereas swimmers near the center focus at the centerline. A further increase in flow rate or swimmer size increases the magnitude of $\mathcal{F}_{\text {passive }}$ relative to wall effects, which results in centerline focusing (region III). For the same reason, as the force-dipole strength decreases [see Fig. 4(b)], the regions of swinging state (region I) and coexisting states (region II) shrink. Pullers, on the other hand, are hydrodynamically repelled from the walls ${ }^{11}$ and therefore always complement $\mathcal{F}_{\text {passive }}$ in centerline focusing [Fig. 3(c) and (d)]. We note that for source-dipole swimmers the hydrodynamic wall interactions are weaker compared to force dipoles since they scale with $\kappa^{3}$, and therefore hardly influence the trajectories. In Table 1, we list expressions for swimming lift and wall interactions that alter the cross-stream migration.

In conclusion, the current study analyzes microswimmers in weakly viscoelastic pressure-driven flows. For neutral and pusher/puller microswimmers, we derive an additional swimming lift velocity depending on the swimmer's hydrodynamic signature that adds to the passive viscoelastic lift. ${ }^{33,41,60,61}$

Table 1 Summary of leading-order contributions in Wi that determine the lift velocity of a microswimmer in the pressure-driven flow of a secondorder fluid. Here, SD and FD refer to source-dipole (neutral squirmer) and force-dipole (pusher/puller) swimmers, respectively. In the third column 1 / $\varepsilon^{n}$ represents the function $\left(\frac{1}{1-x}\right)^{n}-\left(\frac{-1}{1+x}\right)^{n}$

\begin{tabular}{lll}
\hline Type & Lift velocity $\propto$ Wi & Wall contribution to $\dot{x}$ \\
\hline Passive & $\frac{80}{9} x \bar{v}_{\mathrm{m}} \kappa^{2}(1+3 \delta)$ & - \\
SD & $-x(1+\delta) \cos \psi$ & $(\sin \psi) \kappa^{3} / \varepsilon^{3}$ \\
FD & $\frac{8}{3} \kappa \mathcal{P}(1+3 \delta) \sin 2 \psi$ & $(3 / 8) \mathcal{P}\left(1-3 \sin ^{2} \psi\right) \kappa^{2} / \varepsilon^{2}$
\end{tabular}

For source-dipole (neutral) swimmers, the swimming lift is two orders of magnitude stronger than the passive lift, which was considered alone in a recent study. ${ }^{33}$ The current work shows that the swimming lift accelerates the centerline focusing. For force-dipole swimmers (pusher/puller), the swimming lift does not contribute to a net cross-streamline migration. Incorporating hydrodynamic wall interactions, we show that upstream swimming for weak flow strengths qualitatively follows the behavior in Newtonian fluids: ${ }^{11}$ attraction of pushers towards the channel walls and repulsion of pullers. The downstream swimming along the centerline qualitatively remains the same as that of a passive particle. Finally, we stress that our analysis is valid for an arbitrary swimming direction, which can be decomposed in swimming along and perpendicular to the shear plane.

Interestingly, the results suggest that normal stresses in viscoelastic fluids generated by the flow field of a neutral swimmer can accelerate the centerline focusing. However, this strongly depends on the hydrodynamic signature of the microswimmer. Even for a weakly viscoelastic fluid $(\mathrm{Wi}=0.1)$, we observe rapid focusing within a traveled distance of 10-500 times the channel width (Fig. 2), which amounts to ca. 1-50 mm and is quite realistic for microfluidic channels. Thereby, this work contributes to the understanding of swimming in more realistic biological fluids. We note that higher order multipoles like force quadrupole, rotlet dipole and so on, will have their own $\mathcal{F}_{\text {swim }}$. Therefore, we foresee further development in understanding the trajectories of microswimmers that exhibit higher order multipoles in their hydrodynamic disturbance. Furthermore, the current work offers several new directions to explore. For instance, elongated microswimmers, like passive particles, perform Jeffery orbits in sheared Newtonian fluids. ${ }^{62}$ In viscoelastic fluids the flow disturbances from swimming will alter the orientation evolution of these orbits and hence the swimmer dynamics. ${ }^{34}$ The impact of shearthinning fluids is also an interesting outlook, which can be achieved by the use of more detailed rheological models. ${ }^{46}$

\section{Conflicts of interest}

There are no conflicts to declare.

\section{Acknowledgements}

Support from the Alexander von Humboldt Foundation is gratefully acknowledged.

\section{Notes and references}

$\ddagger$ The correction to the drift velocity in $z$-direction is - Wix $\kappa \sin \psi(1+\delta)$, and the correction to rotation is found to be $-2 \mathrm{Wi} \kappa \sin \psi$. We verified that these modifications do not qualitatively alter the dynamics and thus neglect their contributions for simplicity. Details are provided in the ESI. $\dagger$

1 S. S. Suarez and A. Pacey, Hum. Reprod. Update, 2006, 12, 23-37. 
2 R. Levy, D. B. Hill, M. G. Forest and J. B. Grotberg, Integr. Comp. Biol., 2014, 54, 985-1000.

3 A. Persat, C. D. Nadell, M. K. Kim, F. Ingremeau, A. Siryaporn, K. Drescher, N. S. Wingreen, B. L. Bassler, Z. Gitai and H. A. Stone, Cell, 2015, 161, 988-997.

4 J. C. Conrad and R. Poling-Skutvik, Annu. Rev. Chem. Biomol. Eng., 2018, 9, 175-200.

5 M. Jabbarzadeh, Y. Hyon and H. C. Fu, Phys. Rev. E, 2014, 90, 043021.

6 A. E. Patteson, A. Gopinath and P. E. Arratia, Curr. Opin. Colloid Interface Sci., 2016, 100, 86-96.

7 J. P. Celli, B. S. Turner, N. H. Afdhal, S. Keates, I. Ghiran, C. P. Kelly, R. H. Ewoldt, G. H. McKinley, P. So and S. Erramilli, et al., Proc. Natl. Acad. Sci. U. S. A., 2009, 106, 14321-14326.

8 F. Bretherton and N. M. V. Rothschild, Proc. Biol. Sci., 1961, 153, 490-502.

9 J. Hill, O. Kalkanci, J. L. McMurry and H. Koser, Phys. Rev. Lett., 2007, 98, 068101.

10 R. Nash, R. Adhikari, J. Tailleur and M. Cates, Phys. Rev. Lett., 2010, 104, 258101.

11 A. Zöttl and H. Stark, Phys. Rev. Lett., 2012, 108, 218104.

12 A. Zöttl and H. Stark, Eur. Phys. J. E, 2013, 36, 1-10.

13 C.-k. Tung, F. Ardon, A. Roy, D. L. Koch, S. S. Suarez and M. Wu, Phys. Rev. Lett., 2015, 114, 108102.

14 A. J. Mathijssen, N. Figueroa-Morales, G. Junot, É. Clément, A. Lindner and A. Zöttl, Nat. Comm., 2019, 10, 1-12.

15 E. Lauga, The fluid dynamics of cell motility, Cambridge University Press, 2020, vol. 62.

16 E. Lauga, Phys. Fluids, 2007, 19, 083104.

17 X. Shen and P. E. Arratia, Phys. Rev. Lett., 2011, 106, 208101.

18 L. Zhu, E. Lauga and L. Brandt, Phys. Fluids, 2012, 24, 051902.

19 O. S. Pak, L. Zhu, L. Brandt and E. Lauga, Phys. Fluids, 2012, 24, 103102.

20 N. C. Keim, M. Garcia and P. E. Arratia, Phys. Fluids, 2012, 24, 081703.

21 G. J. Elfring and E. Lauga, Complex fluids in biological systems, Springer, 2015, pp. 283-317.

22 J. Sznitman and P. E. Arratia, Complex Fluids in Biological Systems, Springer, 2015, pp. 245-281.

23 G. Li and A. M. Ardekani, J. Fluid Mech., 2015, 784, R4.

24 C. Datt, L. Zhu, G. J. Elfring and O. S. Pak, J. Fluid Mech., 2015, 784, R1.

25 G. Li and A. M. Ardekani, Phys. Rev. Lett., 2016, 117, 118001.

26 G. Li and A. M. Ardekani, Eur. J. Comput. Mech., 2017, 26, 44-60.

27 T. R. Ives and A. Morozov, Phys. Fluids, 2017, 29, 121612.

28 C. Datt, G. Natale, S. G. Hatzikiriakos and G. J. Elfring, J. Fluid Mech., 2017, 823, 675-688.

29 Y. Zhang, G. Li and A. M. Ardekani, Phys. Rev. Fluids, 2018, 3, 023101.

30 A. Zöttl and J. M. Yeomans, Nat. Phys., 2019, 15, 554-558.

31 A. Choudhary, T. Renganathan and S. Pushpavanam, J. Fluid Mech., 2020, 899, A4.
32 J. P. Binagia, A. Phoa, K. D. Housiadas and E. S. Shaqfeh, J. Fluid Mech., 2020, 900, A4.

33 A. J. Mathijssen, T. N. Shendruk, J. M. Yeomans and A. Doostmohammadi, Phys. Rev. Lett., 2016, 116, 028104.

34 M. De Corato and G. D’Avino, Soft Matter, 2017, 13, 196-211. 35 A. M. Ardekani and E. Gore, Phys. Rev. E, 2012, 85, 056309. 36 A. Zhang, W. L. Murch, J. Einarsson and E. S. Shaqfeh, J. Nonnewton. Fluid Mech., 2020, 104279.

37 A. P. Berke, L. Turner, H. C. Berg and E. Lauga, Phys. Rev. Lett., 2008, 101, 038102.

38 D. Smith, E. Gaffney, J. Blake and J. Kirkman-Brown, J. Fluid Mech., 2009, 621, 289-320.

39 A. Karnis and S. Mason, J. Rheol., 1966, 10, 571-592.

40 F. Gauthier, H. Goldsmith and S. Mason, J. Rheol., 1971, 15, 297-330.

41 B. Ho and L. Leal, J. Fluid Mech., 1976, 76, 783-799.

42 D. Li and X. Xuan, Phys. Rev. Fluids, 2018, 3, 074202.

43 A. Choudhary, D. Li, T. Renganathan, X. Xuan and S. Pushpavanam, J. Fluid Mech., 2020, 898, A20.

44 A. Choudhary, T. Renganathan and S. Pushpavanam, Phys. Rev. Fluids, 2021, 6, 036701.

45 L. Leal, J. Nonnewton. Fluid Mech., 1979, 5, 33-78.

46 R. B. Bird, C. F. Curtiss, R. C. Armstrong and O. Hassager, Dynamics of polymeric liquids, fluid mechanics, Wiley, 1987, vol. 1.

47 B. Caswell and W. Schwarz, J. Fluid Mech., 1962, 13, 417-426.

48 L. Leal, J. Fluid Mech., 1975, 69, 305-337.

49 D. L. Koch and G. Subramanian, J. Nonnewton. Fluid Mech., 2006, 138, 87-97.

50 M. Lighthill, Commun. Pure Appl. Math., 1952, 5, 109-118.

51 J. R. Blake, J. Fluid Mech., 1971, 46, 199-208.

52 A. Zöttl and H. Stark, J. Phys.: Condens. Matter, 2016, 28, 253001.

53 H. Lamb, Hydrodynamics, Cambridge, Cambridge University Press, 6th edn, 1975.

54 T. Pedley and J. O. Kessler, Annu. Rev. Fluid Mech., 1992, 24, 313-358.

55 K. Drescher, R. E. Goldstein, N. Michel, M. Polin and I. Tuval, Phys. Rev. Lett., 2010, 105, 168101.

56 K. Drescher, J. Dunkel, L. H. Cisneros, S. Ganguly and R. E. Goldstein, Proc. Natl. Acad. Sci. U. S. A., 2011, 108, 10940-10945.

57 S. Chattopadhyay, R. Moldovan, C. Yeung and X. Wu, Proc. Natl. Acad. Sci. U. S. A., 2006, 103, 13712-13717.

58 I. Minoura and R. Kamiya, Cell Motil. Cytoskeleton, 1995, 31, 130-139.

59 S. E. Spagnolie and E. Lauga, J. Fluid Mech., 2012, 700, 105-147.

60 E. F. Lee, D. L. Koch and Y. L. Joo, J. Nonnewton. Fluid Mech., 2010, 165, 1309-1327.

61 G. D'Avino, F. Greco and P. L. Maffettone, Ann. Rev. Fluid Mech., 2017, 49, 341-360.

62 G. B. Jeffery, Proc. Math. Phys. Eng., 1922, 102, 161-179. 